\title{
THIN-WALLED STRUCTURES
}

\section{SPECIAL ISSUE}

\section{COUPLED INSTABILITIES IN METAL STRUCTURES}

\author{
Guest Editors \\ Joseph Loughlan \\ David H Nash \\ James Rhodes
}

\section{EDITORIAL}

This Special Issue of Thin-Walled Structures is entitled 'Coupled Instabilities in Metal Structures' and it essentially details a selection of papers covering various aspects of coupled instability phenomena in this intriguing research area of structural mechanics. The papers have been selected from the proceedings of the sixth international conference on Coupled Instabilities in Metal Structures, CIMS 2012, held in Glasgow, Scotland, UK on 3-5 December 2012. The conference was organised and run jointly by the Department of Mechanical and Aerospace Engineering at Strathclyde University and the Department of Aeronautical and Automotive Engineering at Loughborough University. The sixth conference in Glasgow follows the very successful and developing series of CIMS conferences held in Timisoara (1992), Liege (1996), Lisbon (2000), Rome (2004) and Sydney (2008) with the seventh CIMS conference now scheduled to be held in North America in 2016.

The coupled buckling and post-buckling interactive failure behaviour of metallic structural systems is an area of research study that has received a great deal of attention over the years and which has made a great many contributions to research advancement in the field of structural mechanics and to the evolution and development of our present day codes of practice pertaining to safe and reliable structural designs. The subject of coupled instabilities is indeed a fascinating and intriguing area of research with numerous and widely varying possibilities and this is clearly reflected in the diversity and quality of the research papers detailed in this Special Issue of TWS.

Thin-walled structures, in particular, are susceptible to the effects of coupled mode interaction whereby local effects due to the thinness of the walls interact with the global behaviour of the structural system. Coupled local-distortional-global/flexural behaviour can be encountered in thin-walled columns or coupled local-lateral buckling may occur in thin-walled beams. The Special Issue deals with various coupled instability phenomena in thin-walled structural systems and highlights the considerable progress and advances made in research, analysis and design in recent years and gives a clear indication of the research trends for the future.

The Coupled Instabilities in Metal Structures Special Issue makes a considerable contribution to our knowledge and understanding of the complex structural mechanics pertaining to coupled phenomena and details the research advances made with particular reference to the stability aspects of thin-walled metal structures. Particular 
emphasis has been paid to the advances made in the analysis and design of structural systems which are associated with stability aspects involving more than one mode of buckling. These works have included studies involving theoretical, numerical and experimental research related to the buckling of metal, notably steel, stainless steel and aluminium structures. The research is reported in considerable detail and the studies made have served to give a deep insight and understanding of coupled instability phenomena.

The papers contained in this TWS Special Issue, provide, collectively, a fairly comprehensive state-of-the-art review of the progresses made in research, development, design and manufacture in recent years pertaining to the coupled instability aspects of thin-walled, light gauge, structural systems. The topical areas of interest include Analytical Developments, Plastic Collapse, Buckling and Failure Aspects of Beams, Cold-Formed Steel Structural Systems, Column local-Flexural Interaction, Finite Element Simulation, GBT and Finite Strip Developments, Local, Distortional and Global Buckling, Stiffened Plate Behaviour and Modal Coupling in Shells. The Special Issue includes significant contributions on the Constrained Finite Strip Method, the ECBL approach and on the Direct Strength Method of Design, all of which serve to provide the latest developments and advances made in recent years in the analysis technology for dealing with coupled structural response.

Although the Special Issue features the coupled response of metal structures, in the main, and of thin-walled metal structures in particular, with a prevalent leaning towards civil engineering applications, the consideration of coupled interaction and its implications on the design of Composite Space and Airframe Structures is also highlighted in some detail. The use of Carbon Fibre Reinforced Plastic, CFRP, is a commonly accepted practice for the airframe designs of today's civil passenger airliners. Indeed, a significant step in the aerospace technology of today is that CFRP is now tending towards $50 \%$ of the whole aircraft structure. The special issue details the state-of-the-art in the advances made as well as the challenges ahead relating to the analysis and design of aerospace composite structures with due consideration being given to the effects of coupled instability phenomena.

Joseph Loughlan

David H Nash

James Rhodes 